\title{
M/M/1 RETRIAL QUEUE WITH COLLISIONS AND WORKING VACATION INTERRUPTION UNDER N-POLICY
}

\author{
Li TAO ${ }^{1}$, Zaiming LIU $^{2}$ AND ZHIZHONG WANG $^{2}$
}

\begin{abstract}
Consider an M/M/1 retrial queue with collisions and working vacation interruption under N-policy. We use a quasi birth and death process to describe the considered system and derive a condition for the stability of the model. Using the matrix-analytic method, we obtain the stationary probability distribution and some performance measures. Furthermore, we prove the conditional stochastic decomposition for the queue length in the orbit. Finally, some numerical examples are presented.
\end{abstract}

Keywords. Retrial, collision, working vacation interruption, N-policy.

Mathematics Subject Classification. 60k25, 90B22.

\section{INTRODUCTION}

Queueing systems with server's vacations have been studied extensively, general models can be found in Tian and Zhang [15]. In 2002, Servi and Finn [12] first introduced a new vacation policy and studied an $M / M / 1$ queue, the server commits a lower service rate rather than completely stopping the service during a vacation, which we call working vacation. Liu et al. [18] obtained the stochastic decompositions in the $\mathrm{M} / \mathrm{M} / 1$ queue with working vacations by the matrix-analytic method. Subsequently, Wu and Takagi [4] generalized results in [12] to an M/G/1 queue. Baba [19] considered a GI/M/1 queue with working vacations by the matrix-analytic method. Recently, Li and Tian [8] investigated the GI/M/1 queue

Received January 8, 2012. Accepted October 26, 2012.

1 School of Science, Shandong University of Technology, Zibo 255049, China.

liltaot@126.com

2 School of Mathematics, Central South University, Changsha 410075, China 
with single working vacation. Furthermore, during the working vacation period, the server can stop the vacation if there are customers at a service completion instant. For the vacation interruption models, Li and Tian [9] first introduced and studied an $\mathrm{M} / \mathrm{M} / 1$ queue with working vacations and vacation interruption. Subsequently, Li et al. [10] analyzed a GI/M/1 queue with working vacations and vacation interruption by the matrix-analytic method. Using the method of a supplementary variable, Zhang and Hou [14] investigated the M/G/1 queue. Baba [20] even studied the $\mathrm{M} / \mathrm{PH} / 1$ queue with working vacations and vacation interruption where the vacation time follows a phase type distribution.

In recent years there have been significant contributions to the retrial queues. Retrial queueing systems are described by the feature that the arriving customers who find the server busy join the retrial orbit to try again for their requests. Choi et al. [1] studied an M/M/1 retrial queue. Martin and Corral [13] investigated an $\mathrm{M} / \mathrm{G} / 1$ retrial queue with liner control policy. Next, Lillo [16] considered a GI/M/1 retrial queue. More models can be found in Artalejo and Corral [6]. Many authors also analyzed the retrial system with collisions, since some networks can be treated as these models. Choi et al. [2] studied a retrial queue with collision arising from unslotted CSMA/CD protocol. Kim [7] analyzed an M/M/1 retrial queue with collision and impatience. Kumar et al. [3] discussed an M/M/1 retrial queue with feedback and collisions. Wu et al. [11] investigated a Geo/G/1 retrial queue with preemptive resume and collisions.

Do [17] first studied an M/M/1 queue with both retrials and working vacations. In this paper, we generalize the model in [17,21], and also consider vacation interruption and collisions. In our model, upon the arrival of requests, if the server is busy, requests are forced to wait in the orbit of infinite size. Assume requests in the orbit try to get service from the server with a constant retrial rate. And, it is more reasonable to assume that the server can stop the vacation at a service completion instant, if the number of customers in the orbit achieves $N$. When a vacation ends, a regular busy period starts only if there are at least $N$ customers in the orbit.

This paper is organized as follows. In Section 1, we introduce the model and obtain the infinitesimal generator. In Section 2, we derive the stability condition and the minimal non-negative solution $R$. Using the matrix-analytic method, the stationary probability distribution is obtained in Section 3. In Section 4, we introduce two random variables and give the conditional stochastic decomposition for the queue length. In Section 5, we obtain some important and interesting performance measures. Some numerical results are presented in Section 6. Finally, Section 7 concludes this paper.

\section{QuAsi BIRTH AND DEATH (QBD) PROCESS MODEL}

In this paper, we consider an $\mathrm{M} / \mathrm{M} / 1$ retrial queue with collisions, working vacations, vacation interruption and $\mathrm{N}$-policy at the same time. Request retrials 
from the orbit of infinite size follow a Poisson process with rate $\alpha$. And, if the server is busy, the retrial customer collides with the customer in service resulting in both being shifted to the orbit. During the normal service period, the server begins a working vacation each time when the system becomes empty, i.e., when the server is free and there is no customer in the orbit. At a service completion instant, if there are at least $N$ customers in the orbit, the server will stop the vacation and come back to the normal working level, which means vacation interruption happens. If the number of customers in the orbit is less than $N$, on the other hand, the server will stay in the working vacation period. Meanwhile, if there are at least $N$ customers in the orbit when a vacation ends, the server switches to the normal busy period, otherwise, the server continues the vacation.

We assume that inter-arrival times, inter-retrial times, service times and vacation times are mutually independent. And, the inter-arrival times, the inter-retrial times, the service times during a normal period, the service times during a vacation period and the vacation times are exponentially distributed with parameters $\lambda, \alpha$, $\mu, \eta$ and $\theta$, respectively.

Let $Q(t)$ be the number of customers in the orbit at time $t$, and $J(t)$ be the state of server at time $t$. There are four possible states of the single server as follows:

(a) the server is on a working vacation at time $t$ and the server is free. When the server is in this state $J(t)=0$.

(b) the server is on a working vacation at time $t$ and the server is busy. If the server is in this state $J(t)=1$.

(c) the server is in a regular busy period at time $t$ and the server is free. When the server is in this state $J(t)=2$.

(d) the server is in a regular busy period at time $t$ and the server is busy. If the server is in this state $J(t)=3$.

Then $\{Q(t), J(t)\}$ is a Markov process with state space

$$
\Omega=\{(k, j), k \geq 0, j=0,1,2,3\}
$$

The system states and one step transitions are shown in Figure 1. Using the lexicographical sequence for the states, the infinitesimal generator can be written as

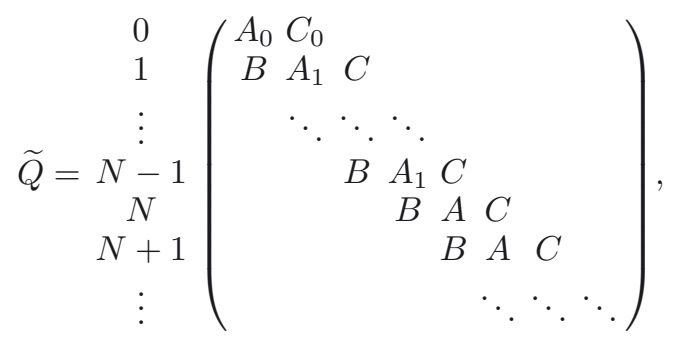




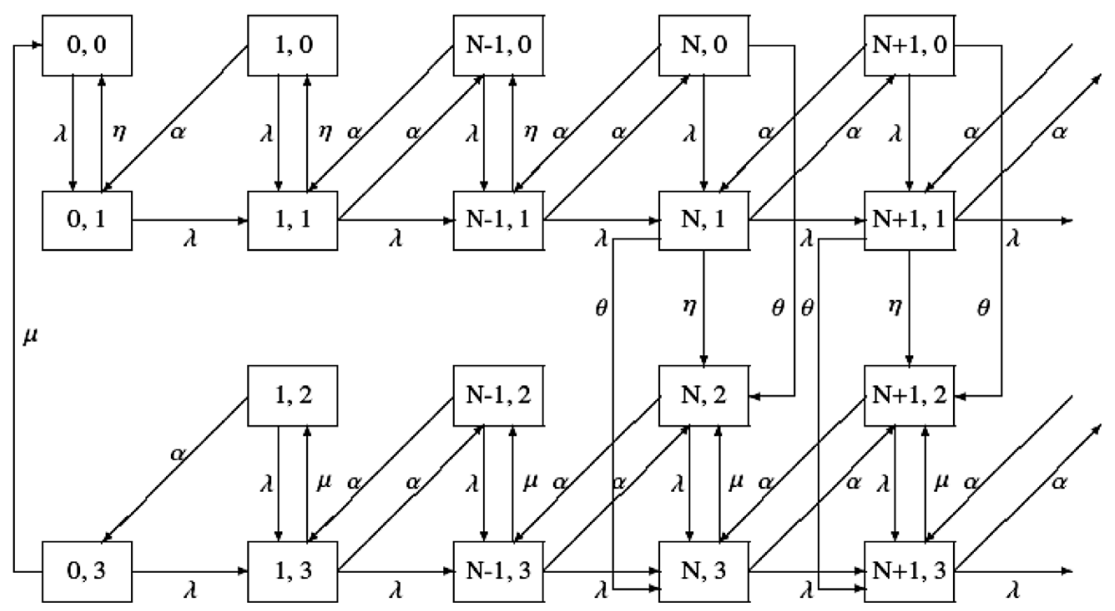

Figure 1. Transition diagram of the states when $\mathrm{N}=3$.

where

$$
\begin{aligned}
& A_{0}=\left(\begin{array}{cccc}
-\lambda & \lambda & 0 & 0 \\
\eta & -\lambda-\eta & 0 & 0 \\
0 & 0 & 0 & 0 \\
\mu & 0 & 0-\lambda-\mu
\end{array}\right) ; \quad C_{0}=\left(\begin{array}{cccc}
0 & 0 & 0 & 0 \\
0 & \lambda & 0 & 0 \\
0 & 0 & 0 & 0 \\
0 & 0 & 0 & \lambda
\end{array}\right) \\
& B=\left(\begin{array}{cccc}
0 & \alpha & 0 & 0 \\
0 & 0 & 0 & 0 \\
0 & 0 & 0 & \alpha \\
0 & 0 & 0 & 0
\end{array}\right) ; \quad A_{1}=\left(\begin{array}{cccc}
-\lambda-\alpha & \lambda & 0 & 0 \\
\eta & -\lambda-\alpha-\eta & 0 & 0 \\
0 & 0 & -\lambda-\alpha & \lambda \\
0 & 0 & \mu & -\lambda-\alpha-\mu
\end{array}\right) ; \\
& A=\left(\begin{array}{cccc}
-\lambda-\alpha-\theta & \lambda & \theta & 0 \\
0 & -\lambda-\alpha-\eta-\theta & \eta & \theta \\
0 & 0 & -\lambda-\alpha & \lambda \\
0 & 0 & \mu & -\lambda-\alpha-\mu
\end{array}\right) ; \quad C=\left(\begin{array}{cccc}
0 & 0 & 0 & 0 \\
\alpha & \lambda & 0 & 0 \\
0 & 0 & 0 & 0 \\
0 & 0 & \alpha & \lambda
\end{array}\right) \text {. }
\end{aligned}
$$

Due to the block structure of matrix $\widetilde{Q},\{Q(t), J(t)\}$ is called a QBD process.

Note that when there is no customer in the orbit, the probability that the server is in a busy period and does not serve a customer is zero.

\section{Stability CONDition AND RATE MATRIX $R$}

In this section, we derive the stability condition and the rate matrix $R$.

Theorem 3.1. The $Q B D$ process $\{Q(t), J(t)\}$ is positive recurrent if and only if $(\mu-2 \lambda) \alpha>\lambda^{2}$. 
Proof. First, we assume

$$
D=B+A+C=\left(\begin{array}{cccc}
-\lambda-\alpha-\theta & \lambda+\alpha & \theta & 0 \\
\alpha & -\alpha-\eta-\theta & \eta & \theta \\
0 & 0 & -\lambda-\alpha & \lambda+\alpha \\
0 & 0 & \alpha+\mu & -\alpha-\mu
\end{array}\right)
$$

Since matrix $D$ is reducible, the Theorem 7.3.1 in [5] gives the condition for positive recurrence of the QBD. After permutation of rows and columns, the Theorem 7.3.1 states that the QBD is positive recurrent if and only if

$$
v\left(\begin{array}{ll}
0 & \alpha \\
0 & 0
\end{array}\right) e>v\left(\begin{array}{ll}
0 & 0 \\
\alpha & \lambda
\end{array}\right) e,
$$

where $e$ is a column vector with all elements equal to one, and $v$ is the unique solution of the system $v\left(\begin{array}{cc}-\lambda-\alpha & \lambda+\alpha \\ \alpha+\mu & -\alpha-\mu\end{array}\right)=\mathbf{0}$, ve $=1$. After some algebraic manipulation, the $\mathrm{QBD}$ process is positive recurrent if and only if $\alpha(\alpha+\mu)>$ $(\lambda+\alpha)^{2}$, i.e., $(\mu-2 \lambda) \alpha>\lambda^{2}$.

Remark 3.2. In order to obtain the stability condition, we can also use the method in [17]. It needs to guarantee that the number of eigenvalues of $Q(x)=$ $B x^{2}+A x+C$ inside the unit disk is 4 . We can easily get that $Q(x)$ have five eigenvalues: $x_{1}=0, x_{2}=\lambda(\lambda+2 \alpha+\theta) /\left[(\lambda+\alpha+\theta)(\lambda+\alpha+\eta+\theta)-\alpha^{2}\right], x_{3}=$ $0, x_{4}=\lambda(\lambda+2 \alpha) / \alpha \mu, x_{5}=1$. Thus, $x_{4}<1$ leads to the result in Theorem 2.1.

Next, we solve the minimal non-negative solution $R$ of the matrix quadratic equation

$$
R^{2} B+R A+C=\mathbf{0}
$$

Theorem 3.3. If $(\mu-2 \lambda) \alpha>\lambda^{2}$, the matrix equation (3.1) has the minimal non-negative solution

$$
R=\left(\begin{array}{cccc}
0 & 0 & 0 & 0 \\
r_{1} & r_{2} & r_{3} & r_{4} \\
0 & 0 & 0 & 0 \\
0 & 0 & r_{5} & r_{6}
\end{array}\right)
$$

where

$$
\begin{aligned}
& r_{1}=\frac{\alpha}{\lambda+\alpha+\theta}, r_{2}=\frac{\lambda(\lambda+2 \alpha+\theta)}{(\lambda+\alpha+\theta)(\lambda+\alpha+\eta+\theta)-\alpha^{2}}, r_{3}=\frac{\theta r_{1}+(\eta+\theta) r_{2}}{\left(1-r_{2}\right) \alpha}, \\
& r_{4}=\frac{\lambda+\alpha}{\mu} r_{3}-\frac{\theta r_{1}+\eta r_{2}}{\mu}, r_{5}=\frac{\lambda+\alpha}{\alpha}, r_{6}=\frac{\lambda(\lambda+2 \alpha)}{\alpha \mu} .
\end{aligned}
$$


Proof. From the structure of $B, A, C$, we can assume $R=\left(\begin{array}{cc}R_{11} & R_{12} \\ 0 & R_{22}\end{array}\right)$, where $R_{11}, R_{12}$ and $R_{22}$ are all $2 \times 2$ matrices. Taking $R$ into (3.1), we have

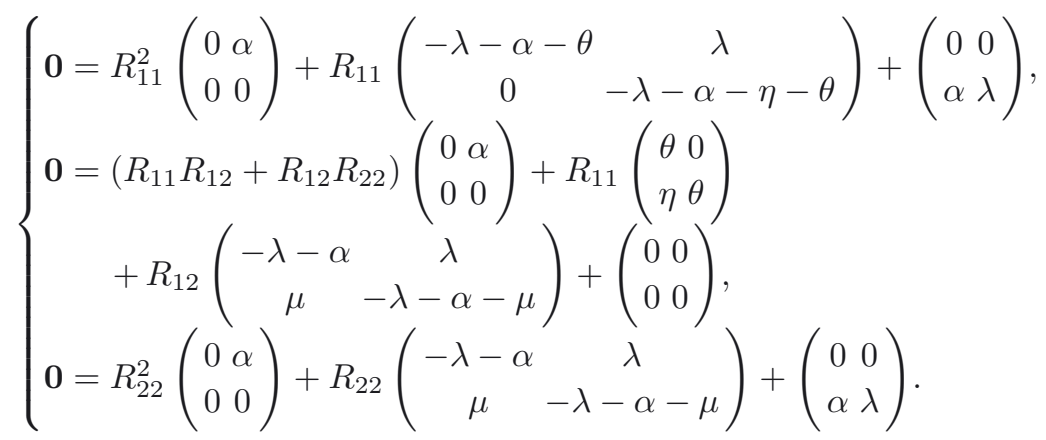

From the first equation, we get $R_{11}=\left(\begin{array}{cc}0 & 0 \\ r_{1} & r_{2}\end{array}\right)$. Similarly, $R_{22}=\left(\begin{array}{cc}0 & 0 \\ r_{5} & r_{6}\end{array}\right)$ can be derived from the third equation. Taking $R_{11}$ and $R_{22}$ into the second equation, we finally obtain $R_{12}=\left(\begin{array}{cc}0 & 0 \\ r_{3} & r_{4}\end{array}\right)$ by some computation.

Remark 3.4. In our model, $B, A, C$ and $\mathrm{R}$ are all block upper triangular matrices. When we solve the equation (3.1), we don't solve it directly, but break the equation into three matrix equations. This method is more effective when the dimension of the matrix is higher, but does not always hold in general case.

\section{Stationary PRobability Distribution}

If $(\mu-2 \lambda) \alpha>\lambda^{2}$, let $(Q, J)$ be the stationary limit of the process $\{Q(t), J(t)\}$, and denote

$$
\begin{aligned}
\pi_{k} & =\left(\pi_{k 0}, \pi_{k 1}, \pi_{k 2}, \pi_{k 3}\right), \quad k \geq 0 \\
\pi_{k j} & =P\{Q=k, J=j\}=\lim _{t \rightarrow \infty} P\{Q(t)=k, J(t)=j\}, \quad(k, j) \in \Omega .
\end{aligned}
$$

Note that from the states we described before, $\pi_{02}=0$.

Theorem 4.1. If $(\mu-2 \lambda) \alpha>\lambda^{2}$, the stationary probability distribution of $(Q, J)$ is given by

$$
\begin{cases}\pi_{k 0}=\pi_{N-1,1} r_{1} r_{2}^{k-N}, & k \geq N, \\ \pi_{k 1}=\pi_{N-1,1} r_{2}^{k+1-N}, & k \geq N, \\ \pi_{k 2}=\pi_{N-1,1}\left(r_{3} r_{2}^{k-N}+\frac{r_{4} r_{5}}{r_{6}-r_{2}}\left(r_{6}^{k-N}-r_{2}^{k-N}\right)\right)+\pi_{N-1,3} r_{5} r_{6}^{k-N}, & k \geq N, \\ \pi_{k 3}=\pi_{N-1,1} \frac{r_{4}}{r_{6}-r_{2}}\left(r_{6}^{k+1-N}-r_{2}^{k+1-N}\right)+\pi_{N-1,3} r_{6}^{k+1-N}, & k \geq N,\end{cases}
$$


and

$$
\begin{aligned}
& \int \pi_{k 0}=\frac{\alpha+\eta}{\lambda+\alpha} \pi_{01}+\frac{\alpha}{\lambda+\alpha}\left(\pi_{11}-\pi_{01}\right) \frac{1-q_{1}^{k-1}}{1-q_{1}}+\frac{\eta}{\lambda+\alpha}\left(\pi_{11}-\pi_{01}\right) \frac{1-q_{1}^{k}}{1-q_{1}}, \\
& 2 \leq k \leq N-2 \text {, } \\
& \pi_{k 1}=\pi_{01}+\left(\pi_{11}-\pi_{01}\right) \frac{1-q_{1}^{k}}{1-q_{1}} \\
& \pi_{k 2}=\frac{\alpha+\mu}{\lambda+\alpha} \pi_{03}+\frac{\alpha}{\lambda+\alpha}\left(\pi_{13}-\pi_{03}\right) \frac{1-q_{2}^{k-1}}{1-q_{2}}+\frac{\mu}{\lambda+\alpha}\left(\pi_{13}-\pi_{03}\right) \frac{1-q_{2}^{k}}{1-q_{2}}, \\
& 2 \leq k \leq N-2 \text {, } \\
& \pi_{k 3}=\pi_{03}+\left(\pi_{13}-\pi_{03}\right) \frac{1-q_{2}^{k}}{1-q_{2}} \\
& \pi_{N-1,0}=\frac{\alpha\left(r_{1} \alpha-\lambda-\alpha-\eta\right)-\lambda \eta}{\lambda \eta+(\lambda+\alpha)\left(r_{1} \alpha-\lambda-\alpha-\eta\right)} \pi_{N-2,1} \\
& \pi_{N-1,1}=\frac{\lambda+\alpha}{\eta} \pi_{N-1,0}-\frac{\alpha}{\eta} \pi_{N-2,1} \\
& \pi_{N-1,2}=r_{3} \pi_{N-1,1}+\frac{\lambda+\alpha}{\alpha} \pi_{N-2,3}, \\
& \pi_{N-1,3}=\frac{\lambda+\alpha}{\mu} \pi_{N-1,2}-\frac{\alpha}{\mu} \pi_{N-2,3},
\end{aligned}
$$

where

$$
q_{1}=\frac{\lambda(\lambda+2 \alpha)}{\alpha \eta}, q_{2}=\frac{\lambda(\lambda+2 \alpha)}{\alpha \mu},
$$

and

$$
\left\{\begin{array}{l}
\pi_{11}=-K^{-1}\left[\frac{\lambda(\lambda+2 \alpha+\eta)}{\lambda+\alpha}+\Delta-K\right] \pi_{01} \\
\pi_{10}=\frac{\eta}{\lambda+\alpha} \pi_{11} \\
\pi_{00}=\frac{\lambda+\eta}{\lambda} \pi_{01}-\frac{\alpha}{\lambda} \pi_{10} \\
\pi_{03}=\frac{\lambda}{\mu} \pi_{00}-\frac{\eta}{\mu} \pi_{01} \\
\pi_{12}=\frac{\lambda+\mu}{\alpha} \pi_{03} \\
\pi_{13}=\frac{\lambda+\alpha}{\mu} \pi_{12}
\end{array}\right.
$$

where $\Delta=\frac{\alpha^{2}\left(r_{1} \alpha-\lambda-\alpha-\eta\right)-\lambda \alpha \eta}{\lambda \eta+(\lambda+\alpha)\left(r_{1} \alpha-\lambda-\alpha-\eta\right)}-\lambda-\alpha-\eta$, and $K=$ $\frac{\lambda(\lambda+2 \alpha)}{\lambda+\alpha} \frac{1-q_{1}^{N-3}}{1-q_{1}}+\left(\Delta+\frac{\lambda \eta}{\lambda+\alpha}\right) \frac{1-q_{1}^{N-2}}{1-q_{1}}$.

Finally, $\pi_{01}$ can be determined by the normalization condition. 
Proof. Using the matrix-geometric solution method (see [5]), we have

$$
\begin{aligned}
\pi_{k} & =\left(\pi_{k 0}, \pi_{k 1}, \pi_{k 2}, \pi_{k 3}\right)=\pi_{N-1} R^{k+1-N} \\
& =\left(\pi_{N-1,0}, \pi_{N-1,1}, \pi_{N-1,2}, \pi_{N-1,3}\right) R^{k+1-N}, \quad k \geq N .
\end{aligned}
$$

And for $k \geq N$,

$$
\begin{aligned}
& R^{k+1-N}= \\
& \left(\begin{array}{cccc}
0 & 0 & 0 & 0 \\
r_{1} r_{2}^{k-N} & r_{2}^{k+1-N} & r_{3} r_{2}^{k-N}+\frac{r_{4} r_{5}}{r_{6}-r_{2}}\left(r_{6}^{k-N}-r_{2}^{k-N}\right) & \frac{r_{4}}{r_{6}-r_{2}}\left(r_{6}^{k+1-N}-r_{2}^{k+1-N}\right) \\
0 & 0 & 0 & 0 \\
0 & 0 & r_{5} r_{6}^{k-N} & r_{6}^{k+1-N}
\end{array}\right)
\end{aligned}
$$

taking $R^{k+1-N}$ into the above equation, we get (4.1). On the other hand, $\pi_{0}, \pi_{1}, \cdots, \pi_{N-1}$ satisfies the next equation

$$
\left(\pi_{0}, \pi_{1}, \cdots, \pi_{N-1}\right) B[R]=\mathbf{0}
$$

where

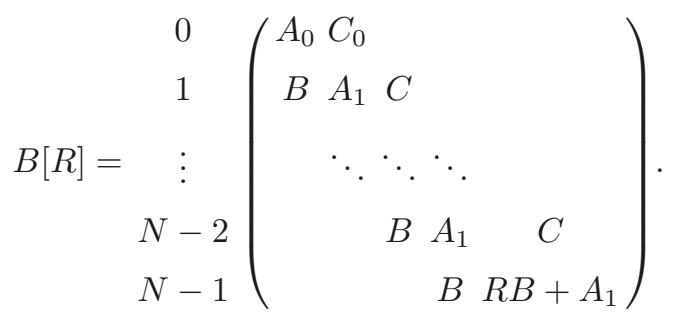

From the infinitesimal generator $\widetilde{Q}$, we can obtain $\pi_{N-2} C+\pi_{N-1} A_{1}+\pi_{N} B=\mathbf{0}$. Since $\pi_{N}=\pi_{N-1} R$, we can get $\pi_{N-2} C+\pi_{N-1}\left(A_{1}+R B\right)=\mathbf{0}$. Thus, we have $R B+A_{1}$ in the last line of $B[R]$.

And,

$$
R B+A_{1}=\left(\begin{array}{cccc}
-\lambda-\alpha & \lambda & 0 & 0 \\
\eta & r_{1} \alpha-\lambda-\alpha-\eta & 0 & r_{3} \alpha \\
0 & 0 & -\lambda-\alpha & \lambda \\
0 & 0 & \mu & r_{5} \alpha-\lambda-\alpha-\mu
\end{array}\right)
$$


Thus, we get

$$
\left\{\begin{array}{l}
-\lambda \pi_{00}+\eta \pi_{01}+\mu \pi_{03}=0 \\
\lambda \pi_{00}-(\lambda+\eta) \pi_{01}+\alpha \pi_{10}=0 \\
-(\lambda+\mu) \pi_{03}+\alpha \pi_{12}=0 \\
-(\lambda+\alpha) \pi_{10}+\eta \pi_{11}=0 \\
-(\lambda+\alpha) \pi_{12}+\mu \pi_{13}=0 \\
\alpha \pi_{k-1,1}-(\lambda+\alpha) \pi_{k 0}+\eta \pi_{k 1}=0 \\
\lambda \pi_{k-1,1}+\lambda \pi_{k 0}-(\lambda+\alpha+\eta) \pi_{k 1}+\alpha \pi_{k+1,0}=0, \quad 1 \leq k \leq N-2 \\
\alpha \pi_{k-1,3}-(\lambda+\alpha) \pi_{k 2}+\mu \pi_{k 3}=0 \\
\lambda \pi_{k-1,3}+\lambda \pi_{k 2}-(\lambda+\alpha+\mu) \pi_{k 3}+\alpha \pi_{k+1,2}=0, \quad 1 \leq k \leq N-2 \\
\alpha \pi_{N-2,1}-(\lambda+\alpha) \pi_{N-1,0}+\eta \pi_{N-1,1}=0, \\
\lambda \pi_{N-2,1}+\lambda \pi_{N-1,0}+\left(r_{1} \alpha-\lambda-\alpha-\eta\right) \pi_{N-1,1}=0 \\
\alpha \pi_{N-2,3}-(\lambda+\alpha) \pi_{N-1,2}+\mu \pi_{N-1,3}=0 \\
\lambda \pi_{N-2,3}+r_{3} \alpha \pi_{N-1,1}+\lambda \pi_{N-1,2}+\left(r_{5} \alpha-\lambda-\alpha-\mu\right) \pi_{N-1,3}=0
\end{array}\right.
$$

From (4.23) and (4.24), we get (4.4) by some computation. Taking (4.4) into (4.23), we get (4.2). In a similar way, we will obtain (4.5) and (4.7) from (4.25) and (4.26). From (4.27) and (4.28), we get $\pi_{N-1,0}$ and $\pi_{N-1,1}$ after some computation. Similarly, taking $r_{5}$ into (4.30), together with (4.29), we can derive $\pi_{N-1,2}$ and $\pi_{N-1,3}$. Then, $\pi_{10}, \pi_{00}, \pi_{03}, \pi_{12}$ and $\pi_{13}$ can be obtained from the equations (4.18)-(4.22). Next, we explain the equation (4.12). Let $k$ take $N-2$ in equation (4.24), using the expressions of $\pi_{N-3,1}, \pi_{N-2,0}, \pi_{N-2,1}$ and $\pi_{N-1,0}$, we get (4.12) after some computation. Since $\sum_{j=0}^{3} \sum_{k=0}^{\infty} \pi_{k j}=1$, we can finally get $\pi_{01}$.

\section{Conditional Stochastic Decomposition}

Consider a retrial $\mathrm{M} / \mathrm{M} / 1$ queue with collisions. This system at any time $t$ can be described by two inter-valued random variables. Let $Q^{*}(t)$ represent the number of customers in the orbit at time $t$, and

$$
J^{*}(t)= \begin{cases}0, & \text { the server is free at time } t \\ 1, & \text { the server is busy at time } t\end{cases}
$$

then $\left\{Q^{*}(t), J^{*}(t)\right\}$ is a Markov process with state space $\{(k, j), k \geq 0, j=0,1\}$. And the infinitesimal generator can be written as

$$
\widetilde{Q}^{*}=\left(\begin{array}{cccccc}
A_{0} & C_{0} & & & \\
B & A & C & & \\
& B & A & C & \\
& & \ddots & \ddots & \ddots
\end{array}\right),
$$


where

$$
\begin{gathered}
A_{0}=\left(\begin{array}{cc}
-\lambda & \lambda \\
\mu & -\lambda-\mu
\end{array}\right) ; C_{0}=\left(\begin{array}{ll}
0 & 0 \\
0 & \lambda
\end{array}\right) ; \\
B=\left(\begin{array}{cc}
0 & \alpha \\
0 & 0
\end{array}\right) ; \quad A=\left(\begin{array}{cc}
-\lambda-\alpha & \lambda \\
\mu & -\lambda-\alpha-\mu
\end{array}\right) ; \quad C=\left(\begin{array}{cc}
0 & 0 \\
\alpha & \lambda
\end{array}\right) .
\end{gathered}
$$

Following the steps we used before, the QBD process $\left\{Q^{*}(t), J^{*}(t)\right\}$ is positive recurrent if and only if $(\mu-2 \lambda) \alpha>\lambda^{2}$. Denote $\tilde{\pi}_{k j}=P\left\{Q^{*}=k, J^{*}=j\right\}=$ $\lim _{t \rightarrow \infty} P\left\{Q^{*}(t)=k, J^{*}(t)=j\right\}$. Then, the stationary probability distribution is given by

$$
\left\{\begin{array}{l}
\widetilde{\pi}_{10}=\frac{\lambda^{2}}{\alpha \mu} \widetilde{\pi}_{00}, \\
\widetilde{\pi}_{k 0}=\widetilde{\pi}_{11} r_{5} r_{6}^{k-2}, \\
\widetilde{\pi}_{01}=\frac{\lambda}{\mu} \widetilde{\pi}_{00}, \\
\widetilde{\pi}_{11}=\frac{\lambda^{2}(\lambda+\alpha)}{\alpha \mu^{2}} \widetilde{\pi}_{00}, \\
\widetilde{\pi}_{k 1}=\widetilde{\pi}_{11} r_{6}^{k-1},
\end{array} \quad k \geq 2,\right.
$$

where $\widetilde{\pi}_{00}$ can be determined by the normalization condition.

Let $Q_{o}=\left\{Q^{*}-1 \mid Q^{*} \geq 1, J^{*}=1\right\}$, and $Q_{o}$ is a conditional queue length given that the server is busy and there are at least one customer in the orbit.

Lemma 5.1. If $(\mu-2 \lambda) \alpha>\lambda^{2}$, then $Q_{o}$ has a probability generating function

$$
G_{Q_{o}}(z)=\frac{1-r_{6}}{1-r_{6} z}
$$

Proof.

$$
\begin{aligned}
G_{Q_{o}}(z) & =\sum_{k=0}^{\infty} z^{k} P\left\{Q_{o}=k\right\}=\sum_{k=0}^{\infty} z^{k} \frac{P\left\{Q^{*}=k+1, J^{*}=1\right\}}{P\left\{Q^{*} \geq 1, J^{*}=1\right\}} \\
& =\sum_{k=0}^{\infty} \frac{\widetilde{\pi}_{11} r_{6}^{k} z^{k}}{\sum_{k=1}^{\infty} \widetilde{\pi}_{11} r_{6}^{k-1}}=\frac{1-r_{6}}{1-r_{6} z} .
\end{aligned}
$$

We introduce a random variable $Q^{N}=\{Q-N \mid Q \geq N, J=1$ or 3$\}$, and $Q^{N}$ is a conditional queue length given that the server is busy and there are at least $N$ customers in the orbit. Let $P_{b}^{*}$ be the probability that the server is busy and there 
are at least $N$ customers in the orbit. Clearly,

$$
\begin{aligned}
P_{b}^{*}= & P\{Q \geq N, J=1 \text { or } 3\}=\sum_{k=N}^{\infty} \pi_{k 1}+\sum_{k=N}^{\infty} \pi_{k 3} \\
= & \sum_{k=N}^{\infty} \pi_{N-1,1} r_{2}^{k+1-N}+\sum_{k=N}^{\infty} \pi_{N-1,1} \frac{r_{4}}{r_{6}-r_{2}}\left(r_{6}^{k+1-N}-r_{2}^{k+1-N}\right) \\
& +\sum_{k=N}^{\infty} \pi_{N-1,3} r_{6}^{k+1-N} \\
= & \frac{r_{4}+r_{2}\left(1-r_{6}\right)}{\left(1-r_{2}\right)\left(1-r_{6}\right)} \pi_{N-1,1}+\frac{r_{6}}{1-r_{6}} \pi_{N-1,3} .
\end{aligned}
$$

Theorem 5.2. If $(\mu-2 \lambda) \alpha>\lambda^{2}$, the conditional queue length $Q^{N}$ can be decomposed into the sum of two independent random variables: $Q^{N}=Q_{o}+Q_{c}$, where $Q_{o}$ follows a geometric distribution with parameter $1-r_{6}$. Additional queue length $Q_{c}$ has a distribution

$$
\begin{aligned}
P\left\{Q_{c}=0\right\} & =\frac{1}{P_{b}^{*}} \frac{\left(r_{2}+r_{4}\right) \pi_{N-1,1}+r_{6} \pi_{N-1,3}}{1-r_{6}}, \\
P\left\{Q_{c}\right. & =k\}=\frac{\pi_{N-1,1}}{P_{b}^{*}} \frac{r_{2}\left(r_{2}+r_{4}-r_{6}\right)}{1-r_{6}} r_{2}^{k-1}, \quad k \geq 1 .
\end{aligned}
$$

Proof. The probability generating function of $Q^{N}$ is as follows:

$$
\begin{aligned}
G_{Q^{N}}(z)= & \sum_{k=0}^{\infty} z^{k} P\left\{Q^{N}=k\right\}=\frac{1}{P_{b}^{*}}\left(\sum_{k=0}^{\infty} z^{k} \pi_{N+k, 1}+\sum_{k=0}^{\infty} z^{k} \pi_{N+k, 3}\right) \\
= & \frac{1}{P_{b}^{*}}\left[\pi_{N-1,1} \frac{r_{2}}{1-r_{2} z}+\pi_{N-1,1} \frac{r_{4}}{\left(1-r_{2} z\right)\left(1-r_{6} z\right)}+\pi_{N-1,3} \frac{r_{6}}{1-r_{6} z}\right] \\
= & \frac{1}{P_{b}^{*}} \frac{1-r_{6}}{1-r_{6} z}\left[\pi_{N-1,1} \frac{r_{2}\left(1-r_{6} z\right)}{\left(1-r_{6}\right)\left(1-r_{2} z\right)}+\pi_{N-1,1} \frac{r_{4}}{\left(1-r_{6}\right)\left(1-r_{2} z\right)}\right. \\
& \left.+\pi_{N-1,3} \frac{r_{6}}{1-r_{6}}\right] \\
= & \frac{1}{P_{b}^{*}} \frac{1-r_{6}}{1-r_{6} z}\left[\frac{\left(r_{2}+r_{4}\right) \pi_{N-1,1}+r_{6} \pi_{N-1,3}}{1-r_{6}}+\pi_{N-1,1} \frac{r_{2}\left(r_{2}+r_{4}-r_{6}\right) z}{\left(1-r_{6}\right)\left(1-r_{2} z\right)}\right] \\
= & \frac{1-r_{6}}{1-r_{6} z}\left[\frac{1}{P_{b}^{*}} \frac{\left(r_{2}+r_{4}\right) \pi_{N-1,1}+r_{6} \pi_{N-1,3}}{1-r_{6}}+\pi_{N-1,1} \frac{1}{P_{b}^{*}} \frac{r_{2}\left(r_{2}+r_{4}-r_{6}\right) z}{\left(1-r_{6}\right)\left(1-r_{2} z\right)}\right] \\
= & G_{Q_{o}}(z) G_{Q_{c}}(z) .
\end{aligned}
$$




\section{Performance measures}

From Theorem 4.1, the probability that the server is busy is

$$
\begin{aligned}
P_{b}= & \sum_{k=0}^{\infty} \pi_{k 1}+\sum_{k=0}^{\infty} \pi_{k 3}=(N-1)\left(\frac{\pi_{11}}{1-q_{1}}-\frac{q_{1} \pi_{01}}{1-q_{1}}\right)-\frac{\pi_{11}-\pi_{01}}{\left(1-q_{1}\right)^{2}}\left(1-q_{1}^{N-1}\right) \\
& +(N-1)\left(\frac{\pi_{13}}{1-q_{2}}-\frac{q_{2} \pi_{03}}{1-q_{2}}\right)-\frac{\pi_{13}-\pi_{03}}{\left(1-q_{2}\right)^{2}}\left(1-q_{2}^{N-1}\right) \\
& +\frac{1-r_{6}+r_{4}}{\left(1-r_{2}\right)\left(1-r_{6}\right)} \pi_{N-1,1}+\frac{1}{1-r_{6}} \pi_{N-1,3},
\end{aligned}
$$

and the probability that the server is free is

$$
P_{f}=\sum_{k=0}^{\infty} \pi_{k 0}+\sum_{k=1}^{\infty} \pi_{k 2}=1-P_{b}
$$

Let $L$ be the number of customers in the orbit, then from Theorem 4.1,

$$
\begin{aligned}
E[L]= & \sum_{k=1}^{\infty} k\left(\pi_{k 0}+\pi_{k 1}+\pi_{k 2}+\pi_{k 3}\right) \\
= & \sum_{k=1}^{N-1} k\left(\pi_{k 0}+\pi_{k 2}\right)+\sum_{k=1}^{N-2} k\left(\pi_{k 1}+\pi_{k 3}\right) \\
& +(N-1) \pi_{N-1,1} \frac{\left(1+r_{1}+r_{3}\right)\left(1-r_{6}\right)+r_{4}\left(1+r_{5}\right)}{\left(1-r_{2}\right)\left(1-r_{6}\right)} \\
& +(N-1) \pi_{N-1,3} \frac{1+r_{5}}{1-r_{6}}+\pi_{N-1,3} \frac{r_{5}+r_{6}}{\left(1-r_{6}\right)^{2}} \\
& +\pi_{N-1,1} \frac{\left(r_{1}+r_{2}+r_{3}\right)\left(1-r_{6}\right)^{2}+r_{4} r_{5}\left(2-r_{2}-r_{6}\right)+r_{4}\left(1-r_{2} r_{6}\right)}{\left(1-r_{2}\right)^{2}\left(1-r_{6}\right)^{2}}
\end{aligned}
$$

Let $L_{s}$ be the number of customers in the system, we have

$$
E\left[L_{s}\right]=\sum_{k=1}^{\infty} k\left(\pi_{k 0}+\pi_{k 2}\right)+\sum_{k=0}^{\infty}(k+1)\left(\pi_{k 1}+\pi_{k 3}\right)=E[L]+P_{b}
$$

Let $W$ be the waiting time of a customer in the orbit, using Little's formula, $E[W]=E[L] / \lambda$. And, the expected sojourn time of a customer in the system $E\left[W_{s}\right]=E\left[L_{s}\right] / \lambda$.

The steady-state interrupted frequency $I F$ of the service due to collisions is given by

$$
I F=\sum_{k=1}^{\infty} \alpha\left(\pi_{k 1}+\pi_{k 3}\right)=\alpha\left(P_{b}-\pi_{01}-\pi_{03}\right)
$$




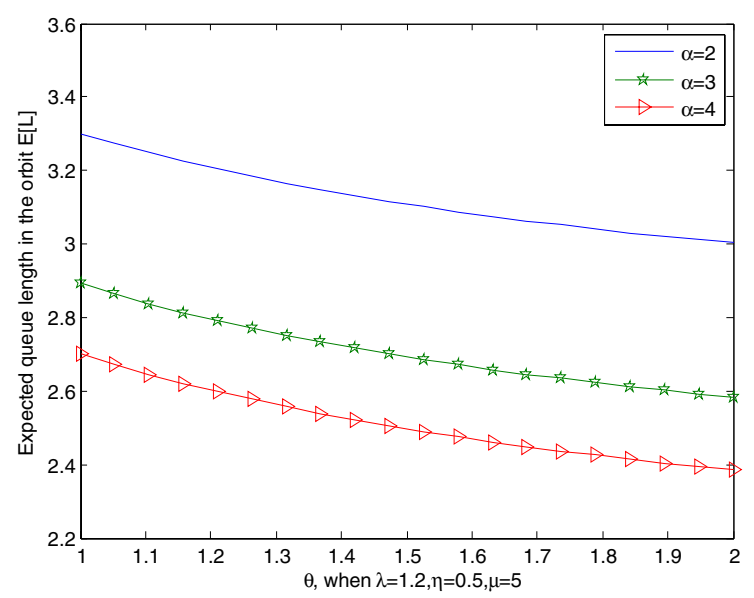

Figure 2. The expected queue length in the orbit with the change of $\theta$.

Let $T$ be the system busy period that starts at an epoch when an arriving customer finds an empty system and ends at the next departure epoch at which the system is empty. Using the theory of regenerative processes,

$$
\pi_{00}=\frac{E\left[T_{00}\right]}{1 / \lambda+E[T]},
$$

where $E\left[T_{00}\right]$ is the amount of time in a regenerative cycle during which the system is in the state $(0,0)$. Clearly, $E\left[T_{00}\right]=1 / \lambda$. Thus, $E[T]=\lambda^{-1}\left(\pi_{00}^{-1}-1\right)$.

\section{Numerical RESUlts}

In this section, taking $N=3, \lambda=1.2$ and $\mu=5$, we present some numerical examples to illustrate the effect of the vacation rate $\theta$, service rate $\eta$ and retrial rate $\alpha$.

Figure 2 illustrates the expected queue length in the orbit $E[L]$ with the change of $\theta$ at different retrial rate $\alpha$. Let $\eta=0.5<\mu=5$, we can find that $E[L]$ decreases with the rate $\theta$ increasing. And, it's easy to see that, if the other conditions are same, the larger retrial rate $\alpha$ is, the smaller $E[L]$ becomes. In Figure 3, with the change of vacation rate $\theta$, the curves of $E\left[L_{s}\right]$ (the expected queue length in the system) and $E\left[W_{s}\right]$ (the expected sojourn time) are provided. $E\left[L_{s}\right]$ and $E\left[W_{s}\right]$ both decrease with an increasing value of $\theta$.

Figure 4 shows the influence of service rate $\eta$ on the expected queue length in the orbit $E[L]$. Taking $\theta=1$, it's obvious that $E[L]$ decreases evidently with the rate $\eta$ increasing. When $\eta=0$, there is no service during the vacation period, so the vacation interruption cannot happen. When service rate $\eta$ approaches to $\mu=5$, the model we considered will become a retrial $\mathrm{M} / \mathrm{M} / 1$ queue with collisions. Under 


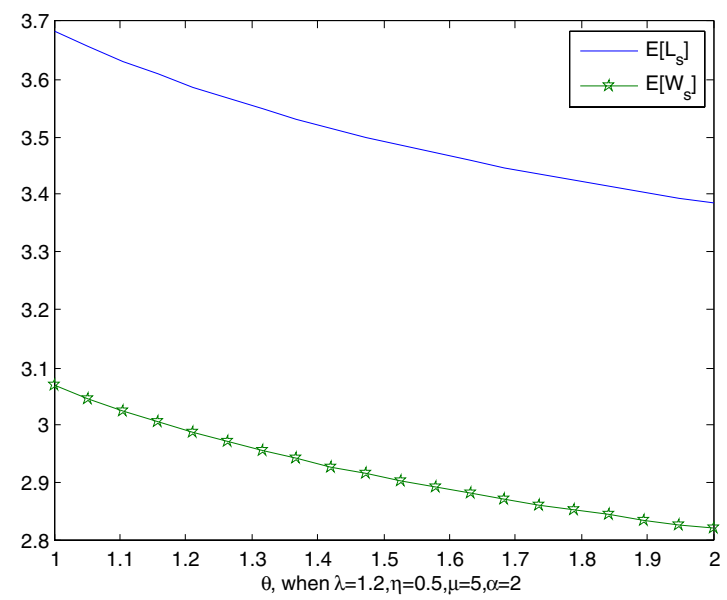

Figure 3. $E\left[L_{s}\right]$ and $E\left[W_{s}\right]$ with the change of $\theta$.

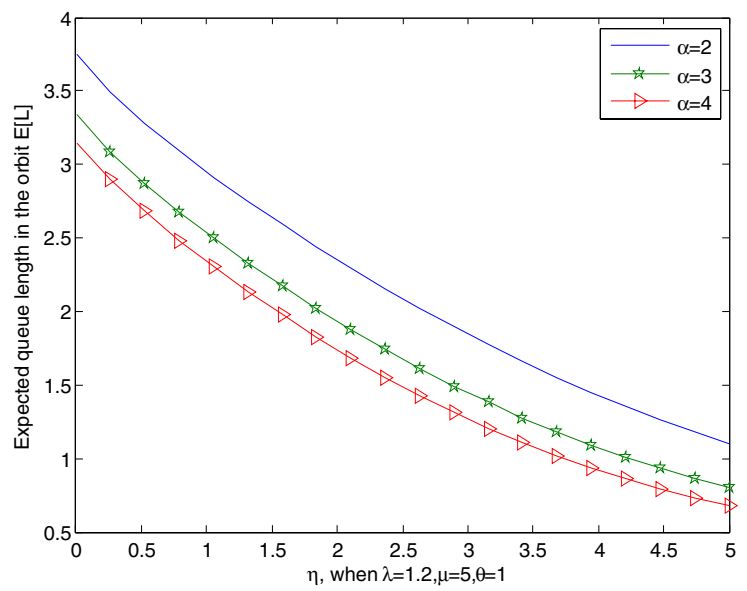

Figure 4. The expected queue length in the orbit with the change of $\eta$.

the vacation policy, if we want to develop a better service, we can consider working vacation policy that utilizes the server and decreases the waiting jobs effectively. In Figure $5, P_{f}$ (the probability that the server is free) and $I F$ (the steady-state interrupted frequency) are plotted versus service rate $\eta$. We find that $P_{f}$ increases as $\eta$ increases while $I F$ decreases as $\eta$ increases.

From Theorem 3.1, we vary the retrial rate $\alpha$ from 2 to 4 . Figure 6 shows the effect of retrial rate $\alpha$ on the expected queue length in the orbit $E[L]$. It's easy to see $E[L]$ decreases with the rate $\alpha$ increasing, this is due to the fact that the interretrial time becomes shorter. And, the larger service rate $\eta$ is, the smaller $E[L]$ 


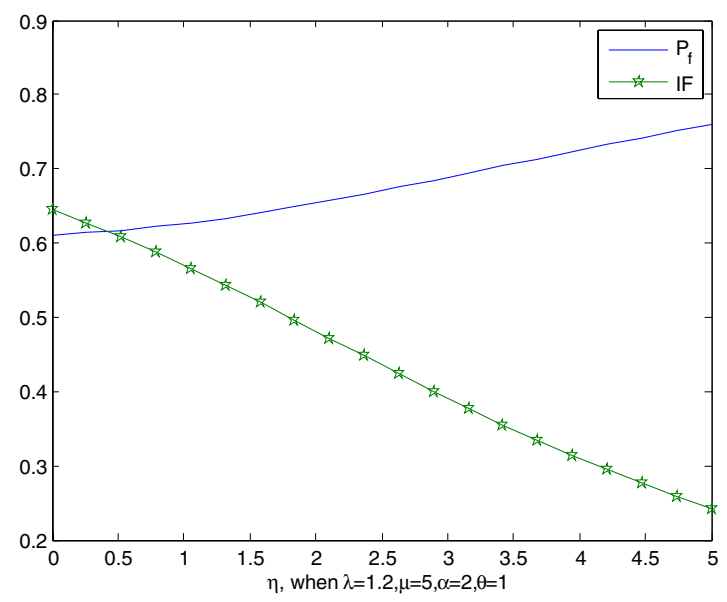

Figure 5. $P_{f}$ and $I F$ with the change of $\eta$.

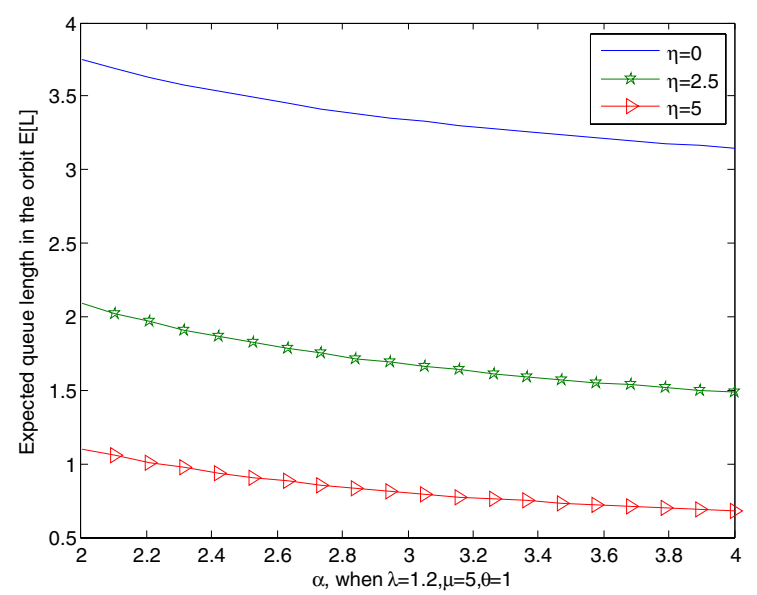

Figure 6 . The expected queue length in the orbit with the change of $\alpha$.

becomes. In Figure 7, the effect of retrial rate on $E[T]$ (the system busy period) and $I F$ are presented. We can see that $E[T]$ decreases evidently with an increasing value of $\alpha$. And as expected, $I F$ increases with the retrial rate $\alpha$ increasing.

\section{Conclusion}

In this paper, an $\mathrm{M} / \mathrm{M} / 1$ retrial queue with collisions and working vacation interruption under N-policy is analyzed. Using the matrix-analytic method, we obtain the stationary probability distribution. We also derive the conditional 


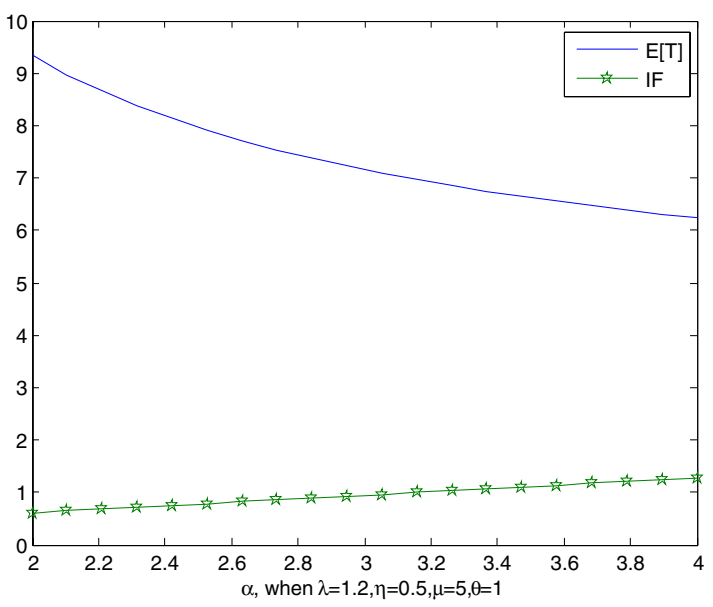

FiguRE 7. $E[T]$ and $I F$ with the change of $\alpha$.

stochastic decomposition and some performance measures. Under the stability condition, we perform some numerical examples to study the effect of various parameters on the system's characteristics. And, the model we considered without vacation interruption can be discussed in a similar way.

\section{Appendix. A}

If the matrix $A=A_{0}+A_{1}+A_{2}$ is reducible, and $\mathrm{A}$ is written, possibly after a permutation of its rows and columns, as

$$
A=\left(\begin{array}{ccccc}
C^{(1)} & 0 & \cdots & 0 & 0 \\
0 & C^{(2)} & \cdots & 0 & 0 \\
\vdots & \vdots & \ddots & \vdots & \vdots \\
0 & 0 & \cdots & C^{(K)} & 0 \\
D^{(1)} & D^{(2)} & \cdots & D^{(K)} & D^{(0)}
\end{array}\right),
$$

where the blocks $C^{(k)}, 1 \leq k \leq K$ are irreducible and satisfy $C^{(k)} e=0$. The matrices $A_{0}, A_{1}$ and $A_{2}$ are similarly structured, and we have that

$$
A_{i}=\left(\begin{array}{ccccc}
C_{i}^{(1)} & 0 & \cdots & 0 & 0 \\
0 & C_{i}^{(2)} & \cdots & 0 & 0 \\
\vdots & \vdots & \ddots & \vdots & \vdots \\
0 & 0 & \cdots & C_{i}^{(K)} & 0 \\
D_{i}^{(1)} & D_{i}^{(2)} & \cdots & D_{i}^{(K)} & D_{i}^{(0)}
\end{array}\right) .
$$

The continuous time case of Theorem 7.3.1 in [5]: Assume that the matrix $A=A_{0}+A_{1}+A_{2}$ is partitioned as in (A.1), where $K \geq 1$ and the matrices $C^{(k)}$, 
$1 \leq k \leq K$ are irreducible. The continuous time QBD is positive recurrent if and only if $\gamma^{(k)} C_{2}^{(k)} e>\gamma^{(k)} C_{0}^{(k)} e$ for all $k: 1 \leq k \leq K$, where $\gamma^{(k)}$ is the unique solution of the system $\gamma^{(k)} C^{(k)}=\mathbf{0}, \gamma^{(k)} e=1$.

Remark A.1. The matrices $A_{0}, A_{1}, A_{2}$ and $A$ represent matrices $C, A, B$ and $D$ in our model, respectively.

\section{REFERENCES}

[1] B. Choi, K. Park and C. Pearce, An M/M/1 retrial queue with control policy and general retrial times. Queueing Syst. 14 (1993) 275-292.

[2] B. Choi, Y. Shin and W. Ahn, Retrial queues with collision arising from unslotted CSMA/CD protocol. Queueing Syst. 11 (1992) 335-356.

[3] B. Kumar, G. Vijayalakshmi, A. Krishnamoorthy and S. Basha, A single server feedback retrial queue with collisions. Comput. Oper. Res. 37 (2010) 1247-1255.

[4] D. Wu and H. Takagi, M/G/1 queue with multiple working vacations. Perform. Eval. 63 (2006) 654-681.

[5] G. Latouche and V. Ramaswami, Introduction to matrix analytic methods in stochastic modelling. ASA-SIAM Series on Applied Probability, USA (1999).

[6] J. Artalejo and A. Corral, Retrial queueing systems. Springer, Berlin (2008).

[7] J. Kim, Retrial queueing system with collision and impatience. Commun. Korean Math. Soc. 25 (2010) 647-653.

[8] J. Li and N. Tian, Performance analysis of a GI/M/1 queue with single working vacation. Appl. Math. Comput. 217 (2011) 4960-4971.

[9] J. Li and N. Tian, The M/M/1 queue with working vacations and vacation interruption. $J$. Syst. Sci. Syst. Eng. 16 (2007) 121-127.

[10] J. Li, N. Tian and Z. Ma, Performance analysis of GI/M/1 queue with working vacations and vacation interruption. Appl. Math. Modell. 32 (2008) 2715-2730.

[11] J. Wu, Z. Liu and Y. Peng, A discrete-time Geo/G/1 retrial queue with preemptive resume and collisions. Appl. Math. Modell. 35 (2011) 837-847.

[12] L. Servi and S. Finn, M/M/1 queue with working vacations (M/M/1/WV). Perform. Eval. 50 (2002) 41-52.

[13] M. Martin and A. Corral, On the M/G/1 retrial queueing system with liner control policy. Top 3 (1995) 285-305.

[14] M. Zhang and Z. Hou, Performance analysis of M/G/1 queue with working vacations and vacation interruption. J. Comput. Appl. Math. 234 (2010) 2977-2985.

[15] N. Tian and Z. Zhang, Vacation queueing models-theory and applications. Springer-Verlag, New York (2006).

[16] R. Lillo, A G/M/1 queue witn exponential retrial. Top 4 (1996) 99-120.

[17] T. Do, M/M/1 retrial queue with working vacations. Acta Inform. 47 (2010) 67-75.

[18] W. Liu, X. Xu and N. Tian, Stochastic decompositions in the M/M/1 queue with working vacations. Oper. Res. Lett. 35 (2007) 595-600.

[19] Y. Baba, Analysis of a GI/M/1 queue with multiple working vacations. Oper. Res. Lett. 33 (2005) 201-209.

[20] Y. Baba, The M/PH/1 queue with working vacations and vacation interruption. J. Syst. Sci. Syst. Eng. 19 (2010) 496-503.

[21] Z. Zhang and $\mathrm{X} . \mathrm{Xu}$, Analysis for the $\mathrm{M} / \mathrm{M} / 1$ queue with multiple working vacations and N-policy. Infor. Manag. Sci. 19 (2008) 495-506. 